\title{
EP-176
}

\section{Surgical outcomes in cystic neoplasm of the pancreas: A 30-year experience in a tertiary care center}

\author{
Amith KUMAR PAKKALA, Bheerappa NAGARI*, Ashish BANSAL, Venu MADHAV THUMMA, \\ G Surya RAMACHANDRA VARMA, Nava KISHORE, Phani KUMAR N \\ Department of Surgical Gastroenterology, Nizam's Institute of Medical Sciences, Hyderabad, India
}

Introduction: Cystic neoplasm of the pancreas is a diverse group of lesions varying from benign to invasive malignant tumours. Though uncommon, they are diagnosed with increased frequency due to the widespread use of cross-sectional imaging for abdominal complaints.

Methods: We analysed this data from a prospectively maintained database from 1990 to 2020 at a tertiary care hospital in Southern India. Patients' demographic profile, tumour distribution, surgical procedures, and perioperative outcomes including complications were studied.

Results: A total of 146 cases underwent surgery for cystic neoplasm of the pancreas from 1990 to 2020. The median age at presentation was 37 years (11 to 85 years). The female:male ratio was 3:1. In our study $93.8 \%$ of the patients were symptomatic at presentation. The most common location was in the body and tail which was 73.9\%. Among the different surgeries performed, 72 (49.3\%) underwent distal pancreatico splenectomy, 39 (26.7\%) underwent pancreaticoduodenectomy and 33 (22.6\%) underwent central pancreatectomy. Laparoscopic/laparoscopic assisted procedures were performed in 17 (11.7\%) patients. On histopathology examination most common lesion was SPEN 83(56.8\%), 29 (19.9\%) were serous cystic neoplasm and 11 (7.5\%) had mucinous cystic neoplasm. Mean hospital stay was $9.2 \pm 4.2$ days. The most common postoperative complication was POPF which was present in 39 (26.7\%) and out of them, 6 were grade B/C (4.1\%). 8 (5.5\%) had postoperative morbidity of Clavien-Dindo grade 3a or more and out of them, 2 (1.4\%) had postoperative mortality.

Conclusions: Cystic neoplasm of the pancreas is most common in the female population. In expert centres, these can be managed with acceptable postoperative morbidity and mortality. 